\title{
Influence of the noradrenergic system on the formation of intrusive memories in women: an experimental approach with a trauma film paradigm
}

\author{
F. Rombold ${ }^{1,2 *}$, K. Wingenfeld ${ }^{1}$, B. Renneberg ${ }^{2}$, J. Hellmann-Regen ${ }^{1}$, C. Otte $^{1}$ and S. Roepke $^{1}$ \\ ${ }^{1}$ Department of Psychiatry and Psychotherapy, Charité - Universitätsmedizin Berlin, Campus Benjamin Franklin, Berlin, Germany \\ ${ }^{2}$ Department of Psychology, Freie Universitaet Berlin, Berlin, Germany
}

Background. Intrusive memories of traumatic events are a core feature of post-traumatic stress disorder but little is known about the neurobiological formation of intrusions. The aim of this study was to determine whether the activity of the noradrenergic system during an intrusion-inducing stressor would influence subsequent intrusive memories.

Method. We conducted an experimental, double-blind, placebo-controlled study in 118 healthy women. Participants received a single dose of either $10 \mathrm{mg}$ yohimbine, stimulating noradrenergic activity, or $0.15 \mathrm{mg}$ clonidine, inhibiting noradrenergic activity, or placebo. Subsequently, they watched an established trauma film which induced intrusions. The number of consecutive intrusions resulting from the trauma film, the vividness of the intrusions, and the degree of distress evoked by the intrusions were assessed during the following 4 days. Salivary cortisol and $\alpha$-amylase were collected before and after the trauma film.

Results. A significant time $\times$ treatment interaction for the number of intrusions and the vividness of intrusions indicated a different time course of intrusions depending on treatment. Post-hoc tests revealed a delayed decrease of intrusions and a delayed decrease of intrusion vividness after the trauma film in the yohimbine group compared with the clonidine and placebo groups. Furthermore, after yohimbine administration, a significant increase in salivary cortisol levels was observed during the trauma film.

Conclusions. Our findings indicate that pharmacological activation of the noradrenergic system during an emotionally negative event makes an impact on consecutive intrusive memories and their vividness in healthy women. The noradrenergic system seems to be involved in the formation of intrusive memories.

Received 14 October 2015; Revised 8 March 2016; Accepted 12 May 2016; First published online 23 June 2016

Key words: Clonidine, intrusions, post-traumatic stress disorder, trauma film paradigm, yohimbine.

\section{Introduction}

Post-traumatic stress disorder (PTSD) can develop after a traumatic stressor and it affects approximately 5.7\% of the general population in the USA (Kessler et al. 2005) and 1.1-2.9\% in Europe (Wittchen et al. 2011). Intrusive symptoms are a core feature of PTSD comprising, inter alia, recurrent, involuntary and intrusive distressing traumatic memories (American Psychiatric Association, 2013).

Rodent and human studies provide cumulative evidence for the involvement of the noradrenergic system

* Address for correspondence: F. Rombold, Department of Psychiatry and Psychotherapy, Charité University of Medicine Berlin, Campus Benjamin Franklin, Hindenburgdamm 30, 12203, Berlin, Germany.

(Email: felicitas.rombold@charite.de)

Parts of the results of this paper have been presented at the 45th

Annual Conference of the International Society of Psycho-

neuroendocrinology (ISPNE). in PTSD neurobiology (Krystal \& Neumeister, 2009; Bailey et al. 2013). For example, female adult PTSD patients who have been sexually abused during childhood show increased 24-h urinary norepinephrine (NE) compared with women who experienced childhood sexual abuse without PTSD diagnosis and nonabused controls (Lemieux \& Coe, 1995). Furthermore, female and male war refugee PTSD patients show increased salivary $\alpha$-amylase (sAA) activity, an indicator of increased noradrenergic activation (Van Stegeren et al. 2006), after awakening compared with healthy controls, and sAA secretion is positively associated with reported PTSD symptoms (Thoma et al. 2012). In addition, pharmacological interventions with the $\alpha 1$ adrenergic antagonist prazosin, which blocks noradrenergic activation, have been shown to reduce trauma-related nightmares compared with placebo in women with PTSD (Taylor et al. 2006).

However, little is known about the neurobiological influences on the formation of intrusions after traumatic stress. The noradrenergic system could be involved, 
as indicated by studies in which a relationship between increased NE and intrusive memories has been found (Southwick et al. 1999). For example, in a study conducted by Southwick et al. (1993), PTSD patients were administered a single dose of yohimbine, an $\alpha_{2}$ selective antagonist that enhances noradrenergic activation, and placebo, each on a separate test day in a double-blind, randomized balanced order. Of the patients, $40 \%$ reported flashbacks in the yohimbine condition, while only $5 \%$ reported flashbacks in the placebo condition during the test day. Furthermore, in male and female PTSD patients, it has been found that the interaction of increased sAA activity and increased salivary cortisol levels during the consolidation of emotionally negative images predicts an increase in intrusive memories (Nicholson et al. 2014).

Sex-specific biological stress mechanisms involved in memory consolidation (Andreano \& Cahill, 2009) might be responsible for the inconsistent sex differences in intrusive memories in men and women (Felmingham et al. 2012; Bryant et al. 2013). For example, women who underwent a cold pressor test before seeing emotionally negative images recalled more negative images after 2 days compared with men (Felmingham et al. 2012). Cortisol levels after the cold pressor test predicted the number of recalled negative images in women, and neutral images in men (Felmingham et al. 2012). In contrast, the interaction of increased sAA activity and increased salivary cortisol levels during the consolidation of emotionally negative images predicts an increase in intrusive memories in men but not in women (Bryant et al. 2013).

The lack of suitable experimental designs to examine the neurobiology of the formation of intrusive memories has so far limited the number of studies. Recently, trauma film paradigms have been shown to induce short-lasting intrusive memories in healthy individuals (e.g. Holmes \& Bourne, 2008). The only study that has, so far, examined associations among salivary cortisol, sAA, and intrusion formation within the trauma film paradigm found a positive correlation between postfilm salivary cortisol levels and the frequency of intrusions, however, only in individuals with increased sAA activity (Chou et al. 2014).

In contrast to the scarce evidence regarding the influence of the noradrenergic stress system on intrusion formation, there are a large number of studies investigating the influence of the noradrenergic system on other aspects of memory and emotional learning in humans (Van Stegeren, 2008; Roozendaal \& McGaugh, 2011; Schwabe et al. 2012). The administration of adrenergic receptor blockers (e.g. propranolol) leads to impaired memory performance for emotional events and stimuli in humans, while pharmacological noradrenergic activation (e.g. yohimbine) during encoding and consolidation enhances memory for emotional stimuli in the majority of studies (Chamberlain \& Robbins, 2013). A positive association between sAA, assessed shortly after the presentation of emotionally arousing pictures, and memory of these pictures 1 week later has been found in healthy participants (Segal \& Cahill, 2009).

In addition to the catecholaminergic system, the hypothalamus-pituitary-adrenal (HPA) axis releases cortisol when it is activated, and has an impact on the formation of emotional memory during encoding and consolidation in healthy individuals (Schwabe et al. 2012; Finsterwald \& Alberini, 2014). For instance, elevated cortisol levels during encoding and consolidation after administration of hydrocortisone improve memory performance for emotionally arousing pictures compared with neutral pictures (Kuhlmann \& Wolf, 2006). Therefore, cortisol levels during trauma might be relevant in the formation of intrusions.

The aim of the current study was to examine the potential influence of noradrenergic activity during traumatic stress (trauma film) in healthy women on the consecutive development of intrusive memories in an experimental design. We hypothesized that noradrenergic activation would lead to an increased number of intrusive memories of the trauma film, more vivid intrusions, and more distressing intrusions of the trauma film compared with placebo and inhibited noradrenergic activity. In addition, it was hypothesized that lower noradrenergic activity would lead to a decreased number of intrusions, decreased vividness and decreased distress of intrusions compared with placebo.

\section{Method}

\section{Participants}

A total of 118 healthy university students were recruited via official university email lists or public postings. As the rape victim in the trauma film is female, the sample was restricted to women to increase homogeneity of our sample. Furthermore, pilot runs with the trauma film including male and female participants had shown that men did not identify themselves with the victim but rather with a male witness. Exclusion criteria included former or present Diagnostic and Statistical Manual of Mental Disorders, 4th edition (DSM-IV) Axis I disorders (assessed by the Structured Clinical Interview for DSM-IV Axis I Disorders; German version of First et al. 1995), physical illnesses, any medication intake (except oral contraceptive), history of sexual abuse or rape, and pregnancy or lactation period. The HCG ULTRA pregnancy test was implemented to exclude 
pregnancy. All women were between 18 and 44 years old (see Table 1) and spoke German on a native level. Out of the 118 participants, four women were excluded: two participants withdrew from the study after having watched the film for several minutes, one participant was excluded due to fatigue during the session after taking clonidine, and one participant was excluded due to missing diary data. This resulted in the final sample size of $n=114$. All participants received financial remuneration (35€).

\section{Procedure}

The randomized, double-blind, placebo-controlled study was conducted at the Department of Psychiatry and Psychotherapy, Campus Benjamin Franklin, Charité - Universitätsmedizin Berlin, and was approved by the local ethics committee. Participants were asked to refrain from smoking, physical exercise, eating, consuming caffeine, and drinking alcohol or other beverages (except for water) at least $1 \mathrm{~h}$ prior to the assessment. Written informed consent was obtained at least $24 \mathrm{~h}$ before the assessment. Each participant was tested on a separate day.

\section{Experimental phase}

Participants were randomly assigned to either clonidine $(0.15 \mathrm{mg})$, inhibiting noradrenergic activity, placebo, or yohimbine $(10 \mathrm{mg})$, stimulating noradrenergic activity. The pills for both drugs as well as the placebo looked identical, hence ensuring that the experimenter was also blind to the experimental condition.

Administration was conducted $60 \mathrm{~min}$ before the trauma film, since peak plasma levels of oral clonidine occur after 60-90 min with an elimination half-life of about 6 to $12 \mathrm{~h}$ (Reid, 1981) and oral yohimbine peaks in plasma after 45-60 min with an elimination half-life of about 0.60 (s.D. $=0.25$ ) h (Owen et al. 1987). Potential effects of the medication and the trauma film on salivary cortisol levels, sAA activity and blood pressure were measured at seven time points during the study. To do so, saliva was collected and blood pressure was measured by an automatic device (BoSo Medicus Uno; Bosch + Sohn, Germany) at baseline, after medication intake, and five times after the trauma film. The Childhood Trauma Questionnaire (CTQ; German version of Bernstein \& Fink, 1998) was utilized to control for differences in experienced childhood events, as childhood trauma has been shown to be associated with noradrenergic responses to stress (Otte et al. 2005) and with an increased risk of PTSD after trauma in adulthood (Breslau et al. 1999). Since PTSD is positively related to pre-trauma trait anxiety (McNally et al. 2011), the trait scale of the State-Trait Anxiety Inventory (German version of Spielberger \& Gorsuch, 1983) was applied. After the assessment, participants completed the intrusion diary for the next 4 days. Participants were instructed to not talk to other potential participants about the content of the study.

\section{Follow-up session}

After 7 days, the diary was returned and The Impact of Event Questionnaire (German version of Horowitz et al. 1979) was applied to evaluate PTSD-related symptoms: intrusion, avoidance and hyperarousal. In order to not inform participants about the trauma film before the experimental phase, they were asked at the end of the study if they had seen the film 'Irréversible' before. After 4 weeks the participants were contacted and The Impact of Event Questionnaire was conducted again via phone. At the end of the study a debriefing was provided via phone and in written form.

\section{Trauma film and intrusion diary}

The trauma film paradigm was adopted, using a film scene which is particularly suitable for evoking shortlasting intrusions in healthy participants and which has been used in previous studies (Weidmann et al. 2009). The scene from the film 'Irréversible' (directed by Gaspar Noé, 2002) was shown to all participants, depicting a scene (14 min, $40 \mathrm{~s})$ in which a woman is raped by a man. The film was shown on a $2 \times 2.5 \mathrm{~m}$ screen. The sound was played through headphones. The female researcher was present while the participant watched the film.

A pencil-and-paper diary (Holmes et al. 2004; Weidmann et al. 2009) was used to record intrusions throughout the following 4 days. Participants were instructed to 'record any spontaneously occurring memory from the trauma film' instantaneously after occurrence. The difference between image- and thought-based memories was explained to the participant. According to Holmes et al. (2004), memories in the diary were specified by spontaneity of appearance, frequency, modality (image, thought based, or both), content, vividness and the degree of distress. Vividness and degree of distress of every single intrusion were rated from 0 ('not at all') to 5 ('a lot') and evaluated by summing up daily ratings. A text message was sent at 21.00 hours daily to remind participants to transfer inscriptions from their pencil-andpaper diary to an identical online diary to assure participation. When participants turned in the diary, the researcher went through every single memory with them and rated whether the respective memory was intrusive or not. Only memories which occurred 
Table 1. Sample characteristics

\begin{tabular}{|c|c|c|c|c|c|}
\hline Characteristic & Clonidine $(n=38)$ & Placebo $(n=38)$ & Yohimbine $(n=38)$ & Statistics & $p$ \\
\hline Mean age, years (s.D.) & $23.3(3.6)$ & $23.1(3.2)$ & $23.4(4.5)$ & $F_{2,111}=0.08$ & 0.93 \\
\hline Intake of oral contraceptives, $n(\%)$ & $19(50.0)$ & $22(57.9)$ & $13(34.2)$ & $\chi_{2}^{2}=4.43$ & 0.11 \\
\hline Current smoker, $n(\%)$ & $8(21.1)$ & $4(10.5)$ & $9(23.7)$ & $\chi_{2}^{2}=2.45$ & 0.29 \\
\hline Menstrual cycle, $n(\%)$ & & & & $\chi_{2}^{2}=1.02$ & 0.62 \\
\hline Follicular phase & $18(50)$ & $18(47.4)$ & $20(58.8)$ & & \\
\hline Luteal phase & $18(50)$ & $20(52.6)$ & $14(41.2)$ & & \\
\hline Mean BMI, kg/m² (S.D.) & $21.2(2.6)$ & $22.4(3.2)$ & $21.8(3.0)$ & $F_{2,110}=1.57$ & 0.21 \\
\hline Mean CTQ (S.D.) & $37.4(9.1)$ & $38.3(10.0)$ & $36.1(6.1)$ & $F_{2,111}=0.66$ & 0.52 \\
\hline Mean STAI-T (S.D.) & $37.2(7.0)$ & $37.6(7.1)$ & $35.7(6.8)$ & $F_{2,111}=0.81$ & 0.45 \\
\hline Participants who had seen the film before, $n(\%)$ & $2(1.8)$ & $4(3.5)$ & $1(0.9)$ & $\chi_{2}^{2}=2.24$ & 0.33 \\
\hline
\end{tabular}

S.D., Standard deviation; BMI, body mass index; CTQ, Childhood Trauma Questionnaire; STAI-T, trait scale of the Trait Anxiety Inventory.

spontaneously and included imagery with vividness and degree of distress $>0$ were considered intrusive and included in the analyses (Ehlers et al. 2004; Arntz et al. 2005). The researchers were trained how to rate the intrusions following a protocol. Further, they were instructed to contact the principal investigator (who was also blind to group membership) in case of uncertainty how to rate the intrusions. This was only the case twice at the beginning of the study. The interrater reliability of this approach proved to be excellent in a study with a similar intrusion assessment (intraclass correlation =0.997; Hagenaars \& Arntz, 2012).

\section{Salivary assessment}

Saliva samples were collected in order to determine salivary free cortisol and SAA (Salivettes ${ }^{\circledR}$, blue cap; Sarstedt). To ensure homogeneous saliva collection from all salivary glands, participants were instructed to move the cotton swab in their mouth in a circular pattern for $1 \mathrm{~min}$ without actively chewing on it (Nater \& Rohleder, 2009). Samples were subsequently stored at $-20^{\circ} \mathrm{C}$ before biochemical analyses were performed in the Neurobiology Laboratory of the Department of Psychiatry, Charité - Universitätsmedizin Berlin. Salivary cortisol was analysed using a commercially available TR-FRET-based, in-house adopted immunoassay (Cisbio International), which was performed in principle according to the manufacturer's instructions (see online Supplementary material for details). Inter- and intra-assay coefficients of variation were below $12 \%$. sAA activity was determined using a modified protocol (see online Supplementary material for details) of a previously published direct $\alpha$-amylase assay (Lorentz et al. 1999). Inter- and intra-assay coefficients of variation were both lower than $10 \%$ for sAA activity.

\section{Statistical analysis}

Statistical analyses were performed using SPSS version 22.0 (USA). Statistical significance refers to a $p$ value $<0.05$. Differences between treatment groups in demographic baseline variables and in the conducted questionnaires during the study were tested by univariate analysis of variance (ANOVA) for continuous data and Pearson's $\chi^{2}$ test for categorical data. For total cortisol, the area under the curve (AUC) was calculated (Pruessner et al. 2003). Pearson's correlation analyses were performed to analyse a possible association between salivary cortisol (AUC), cortisol peak value (peak - baseline), number of intrusions, vividness of intrusions, and degree of distress evoked by intrusions.

Three sets of repeated-measures mixed-design ANOVA were used to test the effects of treatment (between-subjects factor) and time (within-subjects variable with seven levels) on salivary cortisol levels, sAA and blood pressure. Furthermore, ANOVA was conducted to examine the effects of treatment (between-subjects factor) and time (within-subjects variable with four levels) on number of intrusions, vividness, and degree of distress as dependent variables. For repeated-measures ANOVA, $\eta_{p}^{2}$ was used as effect size. In all ANOVAs, homogeneity of variance was assessed by Levene's statistic and sphericity was examined with Mauchly's test. Since the assumption of sphericity was never met, Greenhouse-Geissercorrected $p$ values are reported. For post-hoc comparisons, a Bonferroni correction for multiple comparisons was performed.

An a priori power analysis revealed that a sample size of 105 yields high power (0.95) to detect a small effect size $\left(\eta_{p}^{2}=0.03\right)$ at $p<0.05$ in a three-group design using repeated-measures ANOVAs (G*Power 3.1; Faul et al. 2007). To take drop-outs into account, sample size was set to 118 participants. 


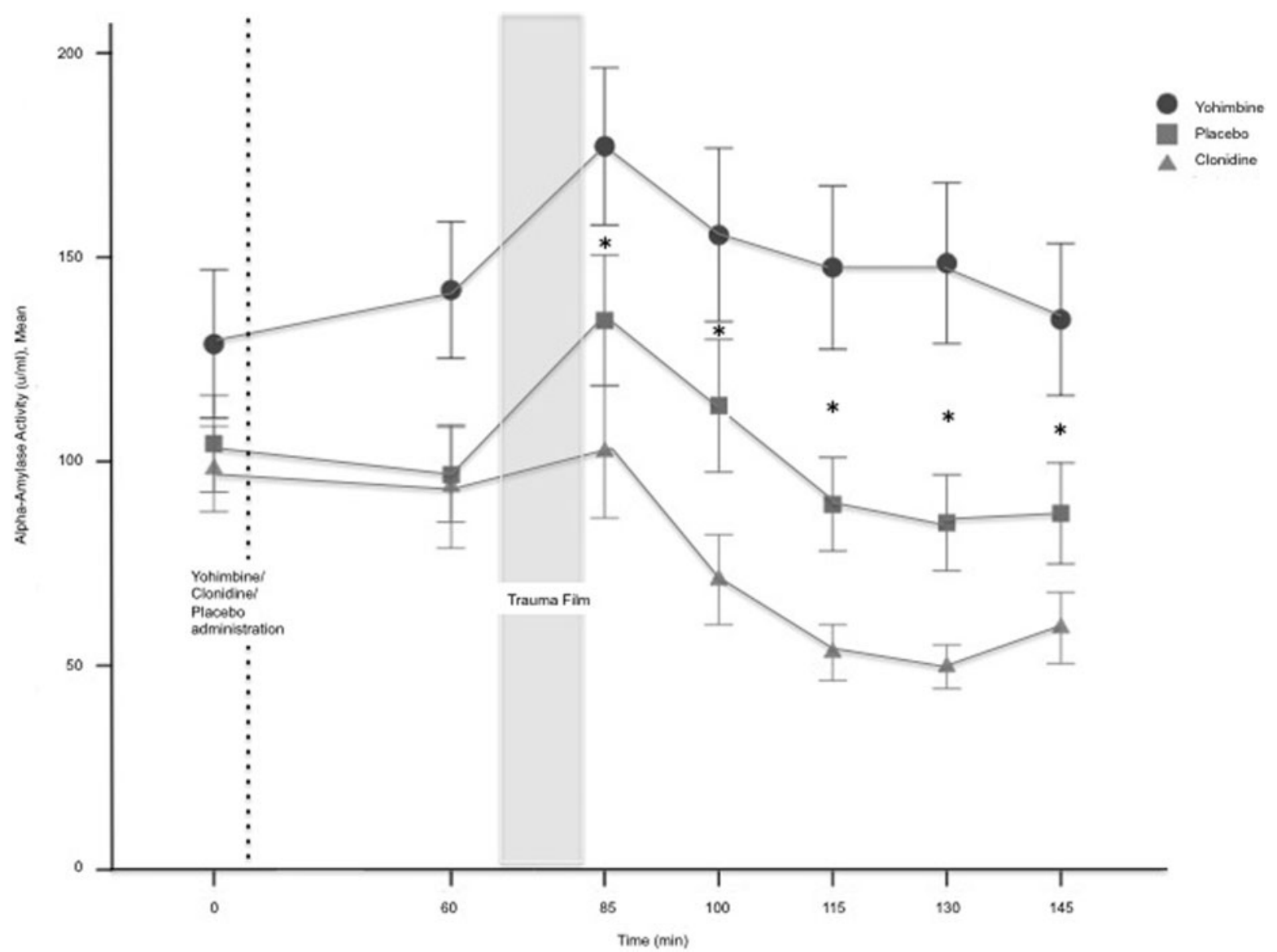

Fig. 1. Salivary $\alpha$-amylase activity at baseline and before and after the trauma film for the clonidine, placebo and yohimbine groups. Values are means, with standard errors represented by vertical bars. * Significant differences between the yohimbine and placebo groups, and the yohimbine and clonidine groups $(p<0.05)$.

\section{Results}

\section{Participant characteristics}

The three groups did not differ in any of the demographic variables or possible confounders (see Table 1).

\section{sAA and blood pressure}

sAA activity throughout the assessment is displayed in Fig. 1 . There was a significant effect of time $\left(F_{3.98,421.71}\right.$ =13.57, $\left.p<0.01, \eta_{p}^{2}=0.11\right)$ as well as a significant effect of treatment $\left(F_{2,106}=7.41, p<0.01, \eta_{p}^{2}=0.12\right)$. Furthermore, a significant time $\mathrm{x}$ treatment interaction $\left(F_{7.96,421.71}=3.14, \quad p<0.01, \quad \eta_{p}^{2}=0.06\right) \quad$ was revealed. Groups did not differ at baseline $(p>0.05)$. Post-hoc tests revealed that yohimbine led to higher sAA activity compared with placebo and clonidine $(p<0.05)$.

Diastolic blood pressure and systolic blood pressure are displayed in Figs 2 and 3. There was a significant effect of time and treatment for diastolic $\left(F_{3.91,430.57}=\right.$ $10.65, p<0.01, \eta_{p}^{2}=0.09, \quad F_{2,110}=33.36, p<0.01, \quad \eta_{p}^{2}=$ $0.38)$ and systolic $\left(F_{4.13,454.52}=16.43, p<0.01, \eta_{p}^{2}=0.13\right.$,
$\left.F_{2,110}=28.42, \quad p<0.01, \eta_{p}^{2}=0.34\right)$ blood pressure. The interaction effect of time $x$ treatment was significant for diastolic $\left(F_{7.83,430.57}=11.16, p<0.01, \eta_{p}^{2}=0.17\right)$ and systolic blood pressure $\left(F_{8.26,454.52}=8.35, p<0.01, \eta_{p}^{2}=\right.$ $0.13)$, indicating that clonidine decreased diastolic $(p<0.01)$ and systolic $(p<0.01)$ blood pressure compared with yohimbine and placebo.

\section{Salivary cortisol}

Salivary cortisol levels for the three experimental groups are displayed in Fig. 4. While there was no treatment effect $\left(F_{2,107}=2.00, p=0.15\right)$, the effect of time was significant $\left(F_{2.00,214.29}=3.52, p<0.01, \eta_{p}^{2}=\right.$ 0.03). Furthermore, the time $x$ treatment interaction was significant $\left(F_{4.01,214.29}=3.37, p=0.01, \eta_{p}^{2}=0.06\right)$. Post-hoc tests revealed no difference in salivary cortisol levels between the three groups at baseline $(p>0.05)$. Furthermore, post-hoc tests revealed higher salivary cortisol levels in the yohimbine group compared with the placebo group $(p<0.05)$ and also compared with the clonidine group on a trend level $(p<0.1)$. 


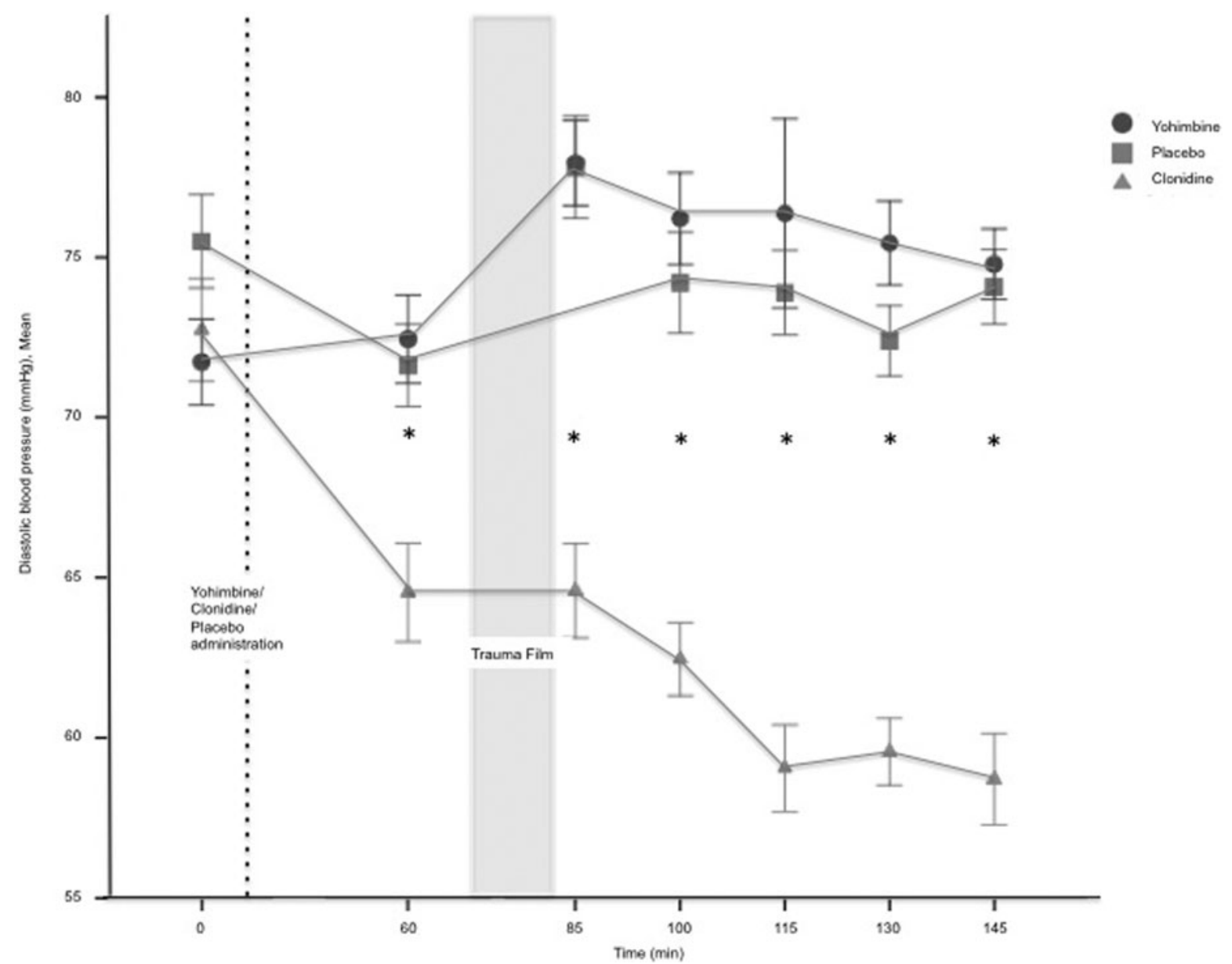

Fig. 2. Diastolic blood pressure at baseline and before and after the trauma film for the clonidine, placebo and yohimbine groups. Values are means, with standard errors represented by vertical bars. * Significant differences between the clonidine and yohimbine groups, and the clonidine and placebo groups $(p<0.01)$.

\section{Intrusions}

As expected, the number of intrusions declined over time $\left(F_{2.47,273.81}=65.24, p<0.01, \eta_{p}^{2}=0.38\right)$. While there was no significant effect of treatment $\left(F_{2,111}=1.00\right.$, $p=0.37)$, a significant time $\mathrm{x}$ treatment interaction was observed $\left(F_{4.93,3.0}=2.97, p=0.01, \eta_{p}^{2}=0.05\right)$, indicating a delayed decrease in the number of intrusive memories in the yohimbine group compared with the clonidine and placebo groups. The interaction is displayed in Fig. 5. Post-hoc tests revealed that participants in the yohimbine group reported more intrusive memories of the trauma film on the first day after the trauma film than participants in the clonidine $(p=0.02)$ and placebo $(p=0.03)$ groups.

As expected, the vividness of intrusive memories also declined over time $\left(F_{2.38,264.55}=32.32, p<0.01\right.$, $\eta_{p}^{2}=0.23$ ). While there was no significant effect of treatment $\left(F_{2,111}=1.34, p=0.27\right)$, the time $\mathrm{x}$ treatment interaction was significant $\left(F_{4.77,264.55}=2.55, p=0.03\right.$, $\eta_{p}^{2}=0.04$ ), indicating a delayed decrease in the vividness of intrusive memories in the yohimbine group compared with the clonidine and placebo groups. The interaction is displayed in Fig. 6. Again, on the first day after the trauma film the reported vividness of the intrusions in the yohimbine group was significantly higher than in the placebo $(p=0.03)$ and clonidine $(p=0.02)$ groups.

Yohimbine, clonidine and placebo did not affect the degree of distress that participants experienced through the intrusive memories (no effect of treatment, $F_{2,111}=1.17, p=0.31$ and no treatment $\mathrm{x}$ time interaction, $\left.F_{4.76,264.40}=1.73, p=0.11\right)$. However, there was a significant effect of time $\left(F_{2.39,264.40}=59.84, p<0.01\right.$, $\left.\eta_{p}^{2}=0.36\right)$, indicating a decline of distress over the course of 4 days.

Including sAA activity and cortisol levels ('AUCs'), childhood trauma (CTQ; German version of Bernstein \& Fink, 1998), or intake of oral contraceptives separately as covariates in the general linear model did not modify the significance of the interaction effect (time $\times$ treatment) for the number of intrusions and 


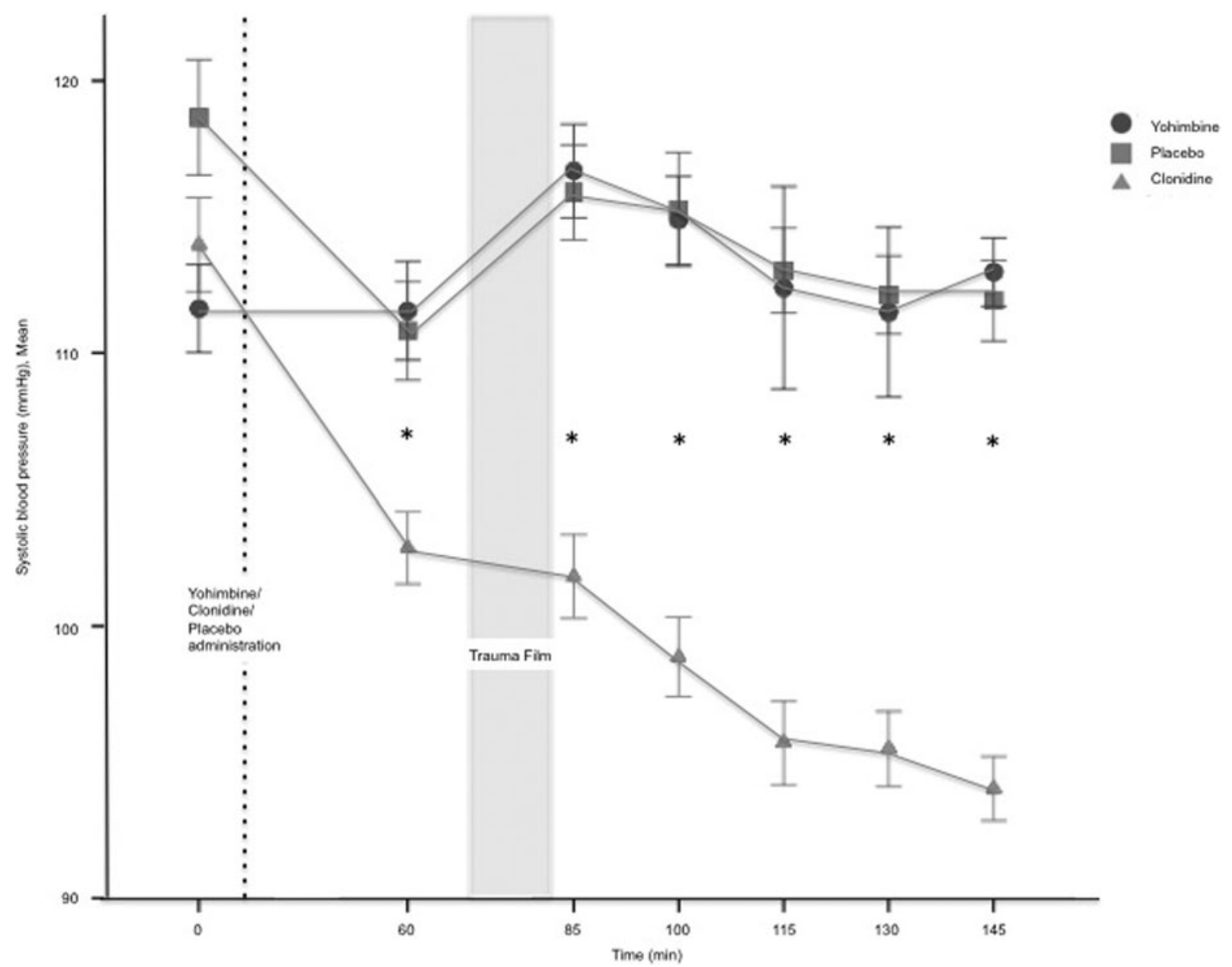

Fig. 3. Systolic blood pressure at baseline and before and after the trauma film for the clonidine, placebo and yohimbine groups. Values are means, with standard errors represented by vertical bars. * Significant differences between the clonidine and yohimbine groups, and the clonidine and placebo groups $(p<0.01)$.

vividness. Further, the number of intrusions, the vividness of intrusions, and the degree of distress evoked by the intrusions were not associated with the cortisol response (AUC and peak value; all $r$ values $<0.1$, all $p$ values $>0.05)$.

\section{The Impact of Event Questionnaire}

The Impact of Event Questionnaire (German version of Horowitz et al. 1979) did not show any differences in PTSD-related symptoms between the yohimbine, clonidine, and placebo groups 1 week $\left(F_{2,108}=0.25, p=0.78\right)$ and 4 weeks after the assessment $\left(F_{2,107}=0.32, p=0.72\right)$.

\section{Discussion}

We examined the influence of the noradrenergic system on encoding and consolidation of potentially intrusive memories after a trauma film paradigm in healthy women. Prior to the trauma film, participants received either yohimbine to stimulate noradrenergic activity, or clonidine to inhibit noradrenergic activity, or placebo. Intrusions occurring throughout the following 4 days were recorded.

After yohimbine the number of intrusive memories as well as their vividness showed a delayed decrease compared with clonidine and placebo over the 4 days following the trauma film. These findings might be relevant for future studies because it has been shown that intrusions during the days and weeks after a trauma predict a PTSD diagnosis later on (Shalev et al. 1996; Ehlers, 2010).

Our results extend recent findings of a study by Bryant et al. (2013), which showed that healthy participants reported more unintentional memories of depicted negative images after being stressed during consolidation compared with non-stressed participants. In this study, an interaction of increased salivary cortisol levels and sAA activity during consolidation predicted more unintentional memories in men but not in women (Bryant et al. 2013). So far, a single study has examined the influence of sAA and salivary 


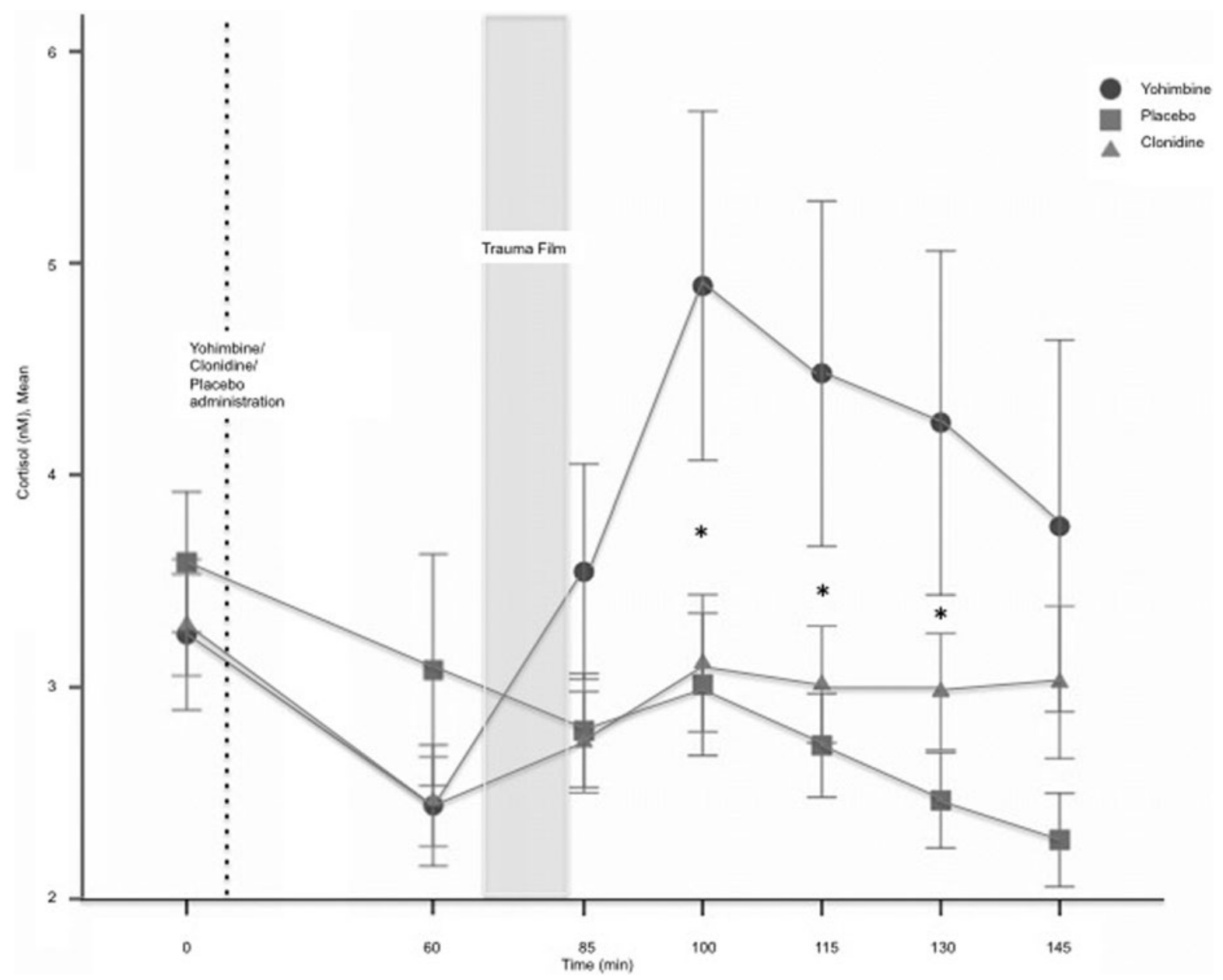

Fig. 4. Salivary cortisol levels at baseline and before and after the trauma film for the clonidine, placebo and yohimbine groups. Values are means, with standard errors represented by vertical bars. * Significant differences between the yohimbine and placebo groups $(p<0.05)$, and difference on a trend level $(p<0.1)$ between the yohimbine and clonidine groups.

cortisol during encoding and consolidation on subsequent intrusions within the trauma film paradigm but without manipulating glucocorticoid and noradrenaline signalling (Chou et al. 2014). However, in this study, sAA activity during encoding and consolidation did not predict the vividness of subsequent intrusions in men and women. Also, peri-film sAA activity was not associated with the number of intrusions. However, a positive correlation between postfilm salivary cortisol levels and the number of intrusions was found in individuals with increased sAA activity (Chou et al. 2014).

A strong increase in salivary cortisol levels $15 \mathrm{~min}$ after the end of the trauma film in the yohimbine condition was found. Contradictory findings exist on the influence of yohimbine administration on salivary cortisol levels in healthy controls. Some findings indicate enhanced salivary cortisol levels after yohimbine administration (Sommer et al. 2011) and others indicate no effect of yohimbine on salivary cortisol levels in controls (Gurguis et al. 1997). In our study, noradrenergic activation after yohimbine during the trauma film might have served as a facilitator of the cortisol response to stress. This, again, might have resulted in the delayed decline of intrusions and their vividness in the yohimbine condition. This mechanism would be in line with findings suggesting that glucocorticoids enhance memory consolidation of emotional stimuli when noradrenergic activity during encoding is increased (Roozendaal et al. 2006). For example, it has been shown that memory for emotional images 1 week after encoding is enhanced by hydrocortisone administration in participants with increased noradrenergic activity at the time of encoding, while this is not the case for participants with hydrocortisone administration but without noradrenergic increase and participants in the placebo condition (Segal et al. 2014).

So far, there are no other experimental studies examining the effects of manipulating the noradrenergic system during encoding and consolidation of stressful or 


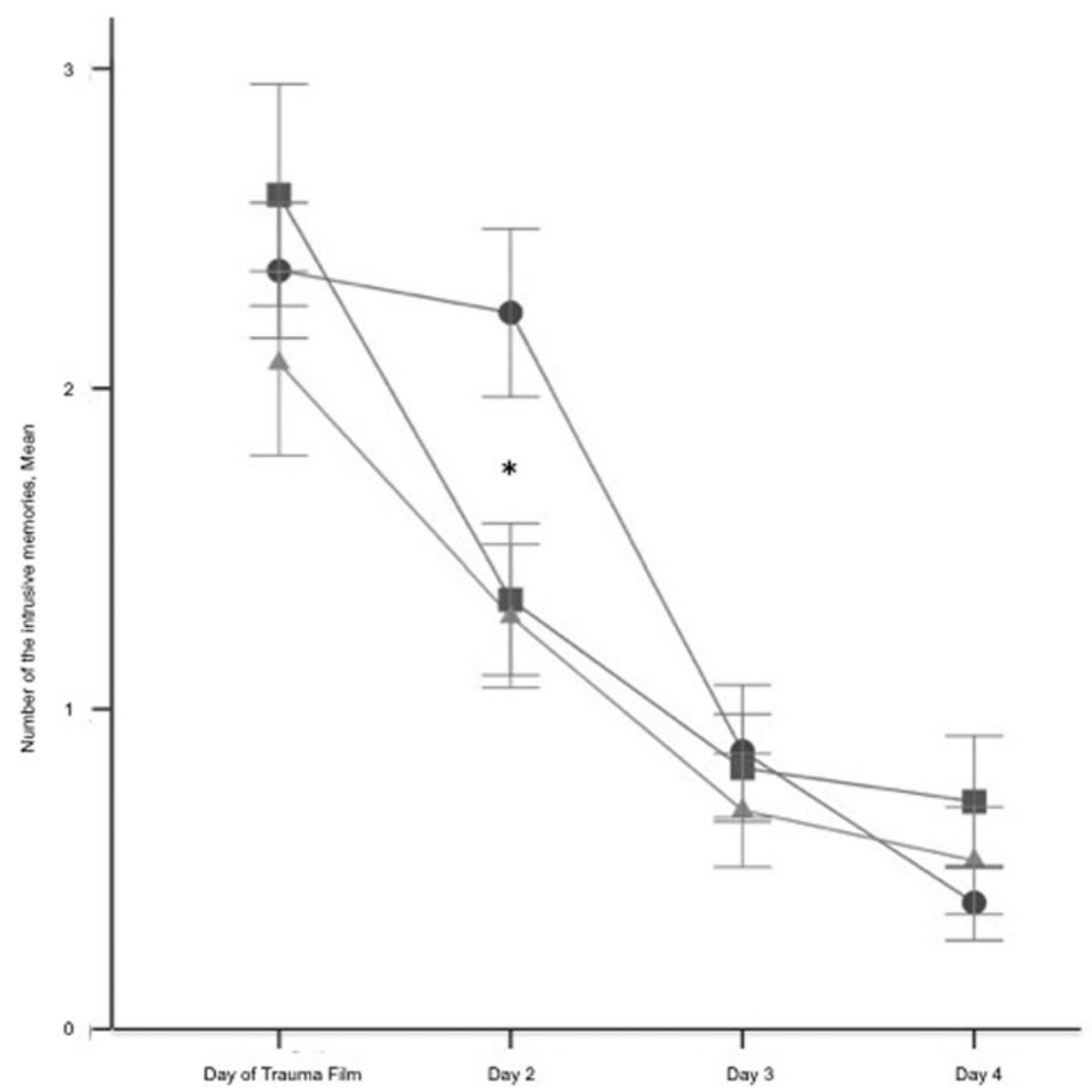

Fig. 5. Number of the intrusive memories for the yohimbine, placebo and clonidine groups over 4 days, starting on the day when the trauma film was shown to the participants. Values are means, with standard errors represented by vertical bars. * Significant differences between the yohimbine and placebo groups, and the yohimbine and clonidine groups $(p<0.05)$.

traumatic events on intrusions. However, our findings seem to be in line with data on stress and emotional memory in rodents and humans. Our results are compatible with data from animal studies suggesting enhanced fear memory after noradrenergic activation during consolidation (Dębiec et al. 2011; Gazarini et al. 2013). Furthermore, the consolidation of emotional events in healthy participants seems to be enhanced by stress (Smeets et al. 2008; Segal \& Cahill, 2009).

No differences in the number of intrusions and their vividness between the clonidine and placebo groups were found. Therefore, noradrenergic inhibition via the $\alpha_{2}$ receptor during trauma does not seem to influence consecutive intrusions, at least in healthy young women and in response to a relatively mild experimental stressor. Several studies examining emotional memories in humans after $\beta$-adrenergic blockade during encoding have reported reduced memory performance for emotional material (Strange et al. 2003; Van Stegeren, 2008). Furthermore, healthy participants show impaired memory in a word list paradigm after administration of a single dose of clonidine during consolidation compared with placebo (Kuffel et al. 2014). In line with these results, a single dose of yohimbine during consolidation after a word list paradigm improves memory performance compared with placebo (Wingenfeld et al. 2013). A mechanism underlying the fact that intrusions did not differ between the clonidine and placebo groups could be the activity of the HPA axis, which did not differ with respect to saliva cortisol between both groups. Furthermore, a floor effect might be responsible for the failure to find an effect of clonidine.

There are some limitations to this study. The sample was restricted to women and female sex hormones may make an impact on intrusive memories (Cheung et al. 2013). Therefore, the results might not be applicable to men. Also, participants taking oral contraceptives were not excluded and the cycle phase was not matched. We did, however, control for both. The lack of effect of clonidine on intrusion formation might be related to the relatively small dosage; however, 


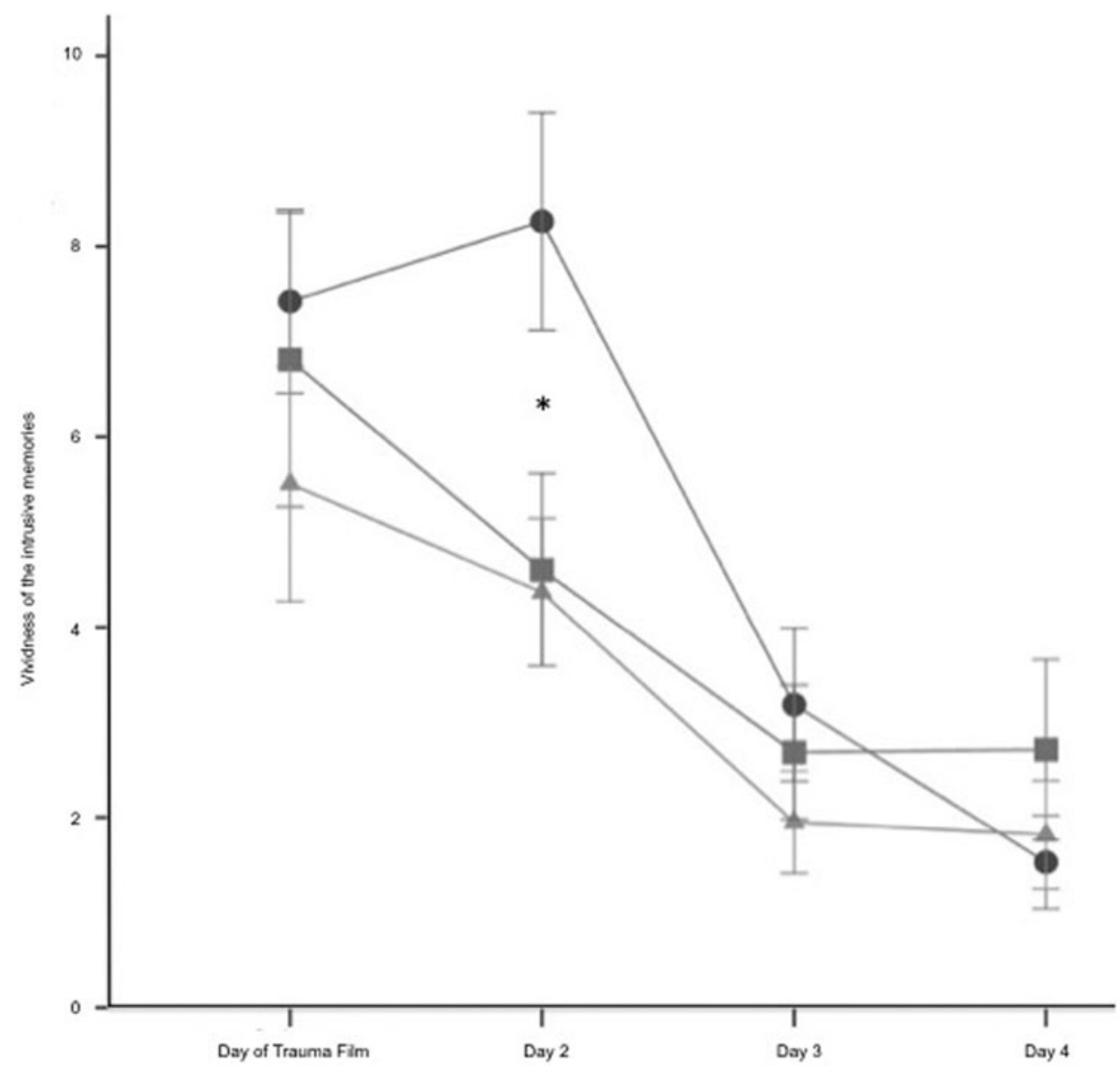

Fig. 6. Vividness of the intrusive memories for the yohimbine, placebo and clonidine groups over 4 days, starting on the day when the trauma film was shown to the participants. Values are means, with standard errors represented by vertical bars. * Significant differences between the yohimbine and placebo groups, and the yohimbine and clonidine groups $(p<0.05)$.

clonidine decreased diastolic and systolic blood pressure compared with yohimbine and placebo, and sedation of the participants at a higher dosage has to be considered. Previous studies have shown that a dosage of $0.15 \mathrm{mg}$ is sufficient to impair memory consolidation in healthy controls (Kuffel et al. 2014). Additionally, a higher dosage $(0.3 \mathrm{mg})$ compared with a lower dosage $(0.15 \mathrm{mg})$ has been shown to have the same effect on the formation of memory in healthy controls (Tiplady et al. 2005). Further, due to the elimination half-life of clonidine and yohimbine, clonidine effects were present during encoding and most of consolidation, whereas yohimbine effects were only present during encoding and early consolidation. Furthermore, in our study design the duration of action of clonidine and yohimbine made it impossible to distinguish between noradrenergic effects during encoding or consolidation of the trauma film on the development of intrusions. This distinction might be especially important regarding future medical treatment interventions, which could more easily be administered during consolidation after the trauma, than during encoding of the traumatic event. Additionally, the decrease of vividness and number of intrusions in the yohimbine group was only of small effect size. The influence of the noradrenergic system on consecutive intrusions after real trauma and clinical significance needs to be elucidated. Furthermore, the self-report of intrusive memories might be inaccurate if the participants fail to recognize the intrusions or do not report them (Takarangi et al. 2014). However, participants' compliance was increased by the daily transfer of recorded intrusions from the paper to the online diary and the reminder per text message.

Future research should focus on investigating the effect of the noradrenergic system and the HPA axis on the formation of intrusions separately by respectively blocking one of the systems. Furthermore, the influence of the noradrenergic system on consecutive intrusions should be evaluated in men and with other trauma film paradigms. In summary, a delayed decrease in intrusive memories as well as a delayed decrease in their vividness after yohimbine administration compared with clonidine and placebo was found. These results 
suggest an influence of noradrenergic activation on intrusion formation. These findings contribute to a better understanding of the neurobiological formation of intrusive memories in healthy individuals without history of trauma exposure. The findings suggest potential mechanisms for animal and human models of PTSD.

\section{Supplementary material}

For supplementary material accompanying this paper visit http://dx.doi.org/10.1017/S0033291716001379

\section{Acknowledgements}

We are grateful for the initial assistance of Dr Anke Weidmann. Further, we are grateful for the assistance of Anna Schmied and Linda Bruch. F.R. was supported by the Elsa-Neumann-Scholarship.

\section{Declaration of Interest}

None.

\section{References}

American Psychiatric Association (2013). Diagnostic and Statistical Manual of Mental Disorders $\left(D S M-5^{\circledR}\right)$. American Psychiatric Association: Washington, DC.

Andreano JM, Cahill L (2009). Sex influences on the neurobiology of learning and memory. Learning and Memory 16, 248-266.

Arntz A, de Groot C, Kindt M (2005). Emotional memory is perceptual. Journal of Behavior Therapy and Experimental Psychiatry 36, 19-34.

Bailey CR, Cordell E, Sobin SM, Neumeister A (2013). Recent progress in understanding the pathophysiology of post-traumatic stress disorder. CNS Drugs 27, 221-232.

Bernstein DP, Fink L (1998). Childhood Trauma Questionnaire: A Retrospective Self-Report: Manual. Psychological Corporation: San Antonio, TX.

Breslau N, Chilcoat HD, Kessler RC, Davis GC (1999). Previous exposure to trauma and PTSD effects of subsequent trauma: results from the Detroit Area Survey of Trauma. American Journal of Psychiatry 156, 902-907.

Bryant RA, McGrath C, Felmingham KL (2013). The roles of noradrenergic and glucocorticoid activation in the development of intrusive memories. PLOS ONE 8, e62675.

Chamberlain SR, Robbins TW (2013). Noradrenergic modulation of cognition: therapeutic implications. Journal of Psychopharmacology 27, 694-718.

Cheung J, Chervonsky L, Felmingham KL, Bryant RA (2013). The role of estrogen in intrusive memories. Neurobiology of Learning and Memory 106, 87-94.

Chou CY, La Marca R, Steptoe A, Brewin CR (2014). Biological responses to trauma and the development of intrusive memories: an analog study with the trauma film paradigm. Biological Psychology 103, 135-143.
Dębiec J, Bush DE, LeDoux JE (2011). Noradrenergic enhancement of reconsolidation in the amygdala impairs extinction of conditioned fear in rats - a possible mechanism for the persistence of traumatic memories in PTSD. Depression and Anxiety 28, 186-193.

Ehlers A (2010). Understanding and treating unwanted trauma memories in posttraumatic stress disorder. Journal of Psychology 218, 141-145.

Ehlers A, Hackmann A, Michael T (2004). Intrusive reexperiencing in post-traumatic stress disorder: phenomenology, theory, and therapy. Memory 12, 403-415.

Faul F, Erdfelder E, Lang AG, Buchner A (2007). G*Power 3: a flexible statistical power analysis program for the social, behavioral, and biomedical sciences. Behavior Research Methods 39, 175-191.

Felmingham KL, Tran TP, Fong WC, Bryant RA (2012). Sex differences in emotional memory consolidation: the effect of stress-induced salivary $\alpha$-amylase and cortisol. Biological Psychology 89, 539-544.

Finsterwald C, Alberini CM (2014). Stress and glucocorticoid receptor-dependent mechanisms in long-term memory: from adaptive responses to psychopathologies. Neurobiology of Learning and Memory 112, 17-29.

First M, Spitzer R, Gibbon M, Williams JB (1995). Structured Clinical Interview for DSM-IV Axis I Disorders (SCID).

New York State Psychiatric Institute, Biometrics Research Department: New York.

Gazarini L, Stern CAJ, Carobrez AP, Bertoglio LJ (2013). Enhanced noradrenergic activity potentiates fear memory consolidation and reconsolidation by differentially recruiting $\alpha 1$ - and $\beta$-adrenergic receptors. Learning and Memory 20, 210-219.

Gurguis GN, Vitton BJ, Uhde TW (1997). Behavioral, sympathetic and adrenocortical responses to yohimbine in panic disorder patients and normal controls. Psychiatry Research 71, 27-39.

Hagenaars MA, Arntz A (2012). Reduced intrusion development after post-trauma imagery rescripting; an experimental study. Journal of Behavior Therapy and Experimental Psychiatry 43, 808-814.

Holmes EA, Bourne C (2008). Inducing and modulating intrusive emotional memories: a review of the trauma film paradigm. Acta Psychologica 127, 553-566.

Holmes EA, Brewin CR, Hennessy RG (2004). Trauma films, information processing, and intrusive memory development. Journal of Experimental Psychology - General 133, 3-21.

Horowitz M, Wilner N, Alvarez W (1979). Impact of Event Scale: a measure of subjective stress. Psychosomatic Medicine 41, 209-218.

Kessler R, Demler O, Frank RG, Olfson M, Pincus HA, Walters EE, Wang P, Kenneth KB, Zaslavsky AM (2005). Prevalence and treatment of mental disorders, 1990 to 2003. New England Journal of Medicine 352, 2515-2523.

Krystal JH, Neumeister A (2009). Noradrenergic and serotonergic mechanisms in the neurobiology of posttraumatic stress disorder and resilience. Brain Research 1293, 13-23.

Kuffel A, Eikelmann S, Terfehr K, Mau G, Kuehl LK, Otte C, Loewe B, Spitzer C, Wingenfeld K (2014).

Noradrenergic blockade and memory in patients with 
major depression and healthy participants.

Psychoneuroendocrinology 40, 86-90.

Kuhlmann S, Wolf OT (2006). Arousal and cortisol interact in modulating memory consolidation in healthy young men. Behavioral Neuroscience 120, 217-223.

Lemieux AM, Coe CL (1995). Abuse-related posttraumatic stress disorder: evidence for chronic neuroendocrine activation in women. Psychosomatic Medicine 57, 105-115.

Lorentz K, Gütschow B, Renner F (1999). Evaluation of a direct $\alpha$-amylase assay using 2-chloro-4-nitrophenyl- $\alpha$-Dmaltotrioside. Clinical Chemistry and Laboratory Medicine 37, 1053-1062.

McNally RJ, Hatch JP, Cedillos EM, Luethcke CA, Baker MT, Peterson AL, Litz BT (2011). Does the repressor coping style predict lower posttraumatic stress symptoms? Military Medicine 176, 752-756.

Nater U, Rohleder N (2009). Salivary $\alpha$-amylase as a non-invasive biomarker for the sympathetic nervous system: current state of research. Psychoneuroendocrinology 34, 486-496.

Nicholson EL, Bryant RA, Felmingham KL (2014). Interaction of noradrenaline and cortisol predicts negative intrusive memories in posttraumatic stress disorder. Neurobiology of Learning and Memory 112, 204-211.

Otte C, Neylan TC, Pole N, Metzler T, Best S, Henn-Haase C, Yehuda R, Marmar CR (2005). Association between childhood trauma and catecholamine response to psychological stress in police academy recruits. Biological Psychiatry 57, 27-32.

Owen J, Nakatsu S, Fenemore J, Condra M, Surridge D, Morales A (1987). The pharmacokinetics of yohimbine in man. European Journal of Clinical Pharmacology 32, 577-582.

Pruessner JC, Kirschbaum C, Meinlschmid G, Hellhammer DH (2003). Two formulas for computation of the area under the curve represent measures of total hormone concentration versus time-dependent change. Psychoneuroendocrinology 28, 916-931.

Reid JL (1981). The Fourth Lilly Prize Lecture, University of Aberdeen, September 1980. The clinical pharmacology of clonidine and related central antihypertensive agents. British Journal of Clinical Pharmacology 12, 295-302.

Roozendaal B, McGaugh JL (2011). Memory modulation. Behavioral Neuroscience 125, 797-824.

Roozendaal B, Okuda S, De Quervain D-F, McGaugh J (2006). Glucocorticoids interact with emotion-induced noradrenergic activation in influencing different memory functions. Neuroscience 138, 901-910.

Schwabe L, Joëls M, Roozendaal B, Wolf OT, Oitzl MS (2012). Stress effects on memory: an update and integration. Neuroscience and Biobehavioral Reviews 36, 1740-1749.

Segal S, Simon R, McFarlin S, Alkire M, Desai A, Cahill L (2014). Glucocorticoids interact with noradrenergic activation at encoding to enhance long-term memory for emotional material in women. Neuroscience 277, 267-272.

Segal SK, Cahill L (2009). Endogenous noradrenergic activation and memory for emotional material in men and women. Psychoneuroendocrinology 34, 1263-1271.

Shalev AY, Peri T, Canetti L, Schreiber S (1996). Predictors of PTSD in injured trauma survivors: a prospective study. American Journal of Psychiatry 153, 219-225.

Smeets T, Otgaar H, Candel I, Wolf OT (2008). True or false? Memory is differentially affected by stress-induced cortisol elevations and sympathetic activity at consolidation and retrieval. Psychoneuroendocrinology 33, 1378-1386.

Sommer M, Braumann M, Althoff T, Backhaus J, Kordon A, Junghanns K, Ehrenthal D, Bartmann U, Hohagen F, Broocks A (2011). Psychological and neuroendocrine responses to social stress and to the administration of the $\alpha$ 2-receptor antagonist, yohimbine, in highly trained endurance athletes in comparison to untrained healthy controls. Pharmacopsychiatry 44, 129-134.

Southwick SM, Bremner JD, Rasmusson A, Morgan CA, Arnsten A, Charney DS (1999). Role of norepinephrine in the pathophysiology and treatment of posttraumatic stress disorder. Biological Psychiatry 46, 1192-1204.

Southwick SM, Krystal JH, Morgan CA, Johnson D, Nagy LM, Nicolaou A, Heninger GR, Charney DS (1993). Abnormal noradrenergic function in posttraumatic stress disorder. Archives of General Psychiatry 50, 266-274.

Spielberger CD, Gorsuch RL (1983). State-Trait Anxiety Inventory for Adults: Manual and Sample: Manual, Instrument and Scoring Guide. Consulting Psychologists Press: Palo Alto, CA.

Strange B, Hurlemann R, Dolan R (2003). An emotioninduced retrograde amnesia in humans is amygdala-and $\beta$ adrenergic-dependent. Proceedings of the National Academy of Sciences of the USA 100, 13626-13631.

Takarangi MK, Strange D, Lindsay DS (2014). Self-report may underestimate trauma intrusions. Consciousness and Cognition 27, 297-305.

Taylor FB, Lowe K, Thompson C, McFall MM, Peskind ER, Kanter ED, Allison N, Williams J, Martin P, Raskind MA (2006). Daytime prazosin reduces psychological distress to trauma specific cues in civilian trauma posttraumatic stress disorder. Biological Psychiatry 59, 577-581.

Thoma MV, Joksimovic L, Kirschbaum C, Wolf JM, Rohleder N (2012). Altered salivary $\alpha$-amylase awakening response in Bosnian War refugees with posttraumatic stress disorder. Psychoneuroendocrinology 37, 810-817.

Tiplady B, Bowness E, Stien L, Drummond G (2005). Selective effects of clonidine and temazepam on attention and memory. Journal of Psychopharmacology 19, 259-265.

Van Stegeren AH (2008). The role of the noradrenergic system in emotional memory. Acta Psychologica 127, 532-541.

Van Stegeren AH, Rohleder N, Everaerd W, Wolf OT (2006). Salivary $\alpha$-amylase as marker for adrenergic activity during stress: effect of betablockade. Psychoneuroendocrinology 31, 137-141.

Weidmann A, Conradi A, Gröger K, Fehm L, Fydrich T (2009). Using stressful films to analyze risk factors for PTSD in analogue experimental studies - which film works best? Anxiety, Stress, and Coping 22, 549-569.

Wingenfeld K, Kuffel A, Uhlmann C, Terfehr K, Schreiner J, Kuehl LK, Otte C, Loewe B, Spitzer C (2013). Effects of noradrenergic stimulation on memory in patients with major depressive disorder. Stress 16, 191-201.

Wittchen H-U, Jacobi F, Rehm J, Gustavsson A, Svensson M, Jönsson B, Olesen J, Allgulander C, Alonso J, Faravelli C (2011). The size and burden of mental disorders and other disorders of the brain in Europe 2010. European Neuropsychopharmacology 21, 655-679. 